\title{
Case Report: Accessory Great Saphenous Vein
}

\author{
Santoshkumar Singarapu ${ }^{1}$, Akashata. B. K ${ }^{1}$, Jeevankumar Giri ${ }^{1}$, \\ Manu Krishnan. ${ }^{1}$, Shobha. G ${ }^{2}$, Uma. B. Gopal ${ }^{3}$ \\ ${ }^{1}$ PG Scholars, ${ }^{2}$ Assistant Professor, ${ }^{3}$ Professor \\ Department of Rachana Shareera, SDM College of Ayurveda and Hospital, Hassan, Karnataka, India
}

\section{ABSTRACT}

The anterior accessory great saphenous vein (AASV) is a common pathway for both primary and recurrent symptomatic varices, which is often inadequately recognized by those unfamiliar with venous anatomy. Varicose veins are a common disorder. Extensive ultrasound (US) mapping of lower extremity chronic venous valvular insufficiency includes the great saphenous vein (GSV), anterior accessory saphenous vein (AASV) and thigh posterior tributary to GSV such as the posterior accessory saphenous vein (PTSV, PASV)(1-3) Anatomically, the AASV vessel rests within the same layer of the limb as the GSV, lying just superficial to the deep venous system over the proximal anterior thigh. It has been shown that both the refluxing GSV and AASV patterns adversely affect patient-perceived quality of life measures.4,5 Despite the common finding of AASV reflux, the majority of governing bodies focus on few vein patterns, namely the great saphenous and small saphenous veins.6Conclusion: Patterns of reflux from the saphenofemoral junction to either the anterior accessory saphenous vein or great saphenous vein possess similar disease severity and commonly suffer complications of venous stasis. The lateral accessory saphenous vein is the commonest cause of recurrence in the groin of varicose veins. It should be looked for specifically during pre-operative assessment duplex scanning and at primary surgery. If identified at operation, we believe it should be either stripped or avulsed to reduce the risk of recurrence.

Keyword: The great saphenous vein (GSV), Anterior accessory saphenous vein (AASV) posterior tributary saphenous vein, posterior accessory saphenous vein (PTSV, PASV)

\section{INTRODUCTION}

The venous system has frequent anatomical variations, so it is more variable compared to the arterial system. A good understanding of the venous anatomy is very important and proper evaluation of the venous system is crucial to the appropriate treatment of venous disorders. Greater saphenous vein (GSV), also known as the vena saphena magna is the longest vein in the body.

The accessory saphenous vein is linked to the greater saphenous vein, which runs parallel to it, and is located in the leg and thigh. The greater saphenous vein is a large superficial (near the skin's surface) vein that originates where the foot's dorsal venous arch merges with the big toe's dorsal vein. It travels up the leg's medial side (closer to the middle) after passing anteriorly to the medial malleolus, a bony projection on the inside of each ankle. The accessory saphenous vein joins it immediately before it drains into the femoral vein. When the accessory saphenous vein reaches the knee, it travels posteriorly along the inner parts of the knee and thigh and finally across the posterior border of the femur bone's medial epicondyle, a rounded bony projection at the lower (distal) end of the femur where it connects to the knee. The accessory saphenous vein then travels to the thigh's front surface before entering the saphenous hiatus, an oval-shaped opening in the fascia lata of the thigh. The fascia lata is a layer of fibrous connective tissue that envelops the thigh muscles. The vein then attaches to the femoral vein in the sapheno femoral junction of the femoral triangle. The femoral triangle is a depression in the upper part of the inner thigh that contains the femoral nerve, artery, and veins as well as lymph nodes and vessels. The greater accessory saphenous vein branches out into smaller veins that are made of elastic fiber. These veins have diminished 
muscle cells and thin walls. They are often the cause of varicose veins, which develop when they dilate.

\section{Case Report:}

During the routine classes of the cadaver dissection of the lower limb in dissection hall of Department of the Rachana Sharira (Anatomy) SDM College Of Ayurveda , Hassan , Karnataka. Observation was made:

1. The lower extremity dissection carried out, the left GSV drained into the superficial femoral vein (SFV) instead of the CVF.

2. There was a short and blind ending vascular structure at the medial aspect of the CFV at the level of the SFJ (fig 01).

3. The diameter of the GSV was $6.3 \mathrm{~mm}$ above and $3.0 \mathrm{~mm}$ below the knee and GSV was insufficient in both locations.

4. There were varicose venous structures up to $9 \mathrm{~mm}$ connecting with GSV both below and above the knee.

5. Perforating veins were detected extending the incision till below the knee i.e head of the tibia, and fallowed till the tip of the great toe i.e course of the great sephanous vein, the erect position between both the GSV and crural veins in the crural region and small saphenous vein and crural veins in the crural region.

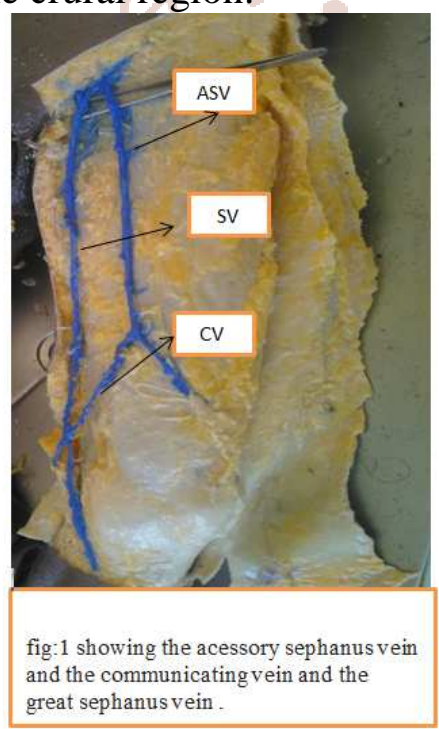

\section{Discussion:}

The GSV is still the most popular vein graft in coronary artery bypass surgery even though synthetic grafts and arteriamammariainterna are becoming used more frequently. It plays an important role in the venous return of the lower extremity because it has a lot of branches along its course and it has relations with deeper veins. The main tributaries are the superficial epigastric vein (SEV), the external pudendal vein (EPV), the superficial circumflex iliac vein (SCIV), the medial accessory saphenous vein (MASV) and the lateral accessory saphenous vein (LASV) . GSV is not only used in coronary bypass surgery but also in the treatment of cerebrovascular diseases and as grafts in peripheric vascular surgery. There are many anatomical variations of the GSV at the level of the thigh, calf and knee. Studies of SFJ began at the turn of the 19th century and were dominated by surgeons who stripped the GSV during surgical treatment of varicose veins. In 1916 Homans was the first to ligate all the tributaries of GSV near the SFJ. This mode of operation, including many different modifications, remains a principal surgical procedure today. Familiarity with the anatomy of this tributary network, termed by Bruska and Hilty theconfluensvenosussubinguinalis, is therefore very important in the radical surgery of the GSV. The confluensvenosussubinguinalis is formed by 5 main veins: GSV, EPV, SEV common truncus, SCIV, and LASV. The connections between them are, however, variable according to Orsini, who distinguished 135 anatomical variants of the GSV, although without classifying them into general groups as Kocon and Zebrowski's study. Konrad identified 5 types of draining pattern of the GSV according to the number of its direct tributaries. Type 1, with 3 direct collaterals of the GSV, is the most frequently encountered (47.8\%). The common trunk, which drains into the GSV, is formed by the conjunction of 2 or 3 collaterals. It is most often formed by 2 tributaries, SEV and SCIV [8]. In our case GSV did not end in the CFV but drained in to the SFV. To our knowledge, no similar case of SFJ anatomical variation has been defined. There have been revolutionary developments in the diagnosis and treatment of chronic venous insufficiency in recent years. The treatment options for varicose veins include conservative approach, sclerotherapy, ultrasound-guided sclerotherapy, and junction ligation with or without vein stripping. Although expertise in venous anatomy and variations may not be necessary in conservative approach, interventional treatment modalities may necessitate expertise. Hence, if important anatomical variations are not recognized, surgical or lesser invasive procedures might result in incomplete SFJ surgery. Consequently the knowledge and identification of anatomical variations play an important role in increasing the success and efficacy of surgical treatments and in decreasing the recurrence rates. 
From descriptions and from the histology of the veins in the saphenous compartment as compared with tributaries, it may be concluded that only veins which course through the saphenous compartment really correspond to the great saphenous vein. Duplication of the great saphenous vein is therefore only present when both vessels course through the compartment. This is in contrast to the considerably higher figures published previously for duplication (27\%, Kubik 1985 in Appendix 2).

\section{Conclusion:}

The great saphenous vein (v. saphena magna; internal or long saphenous vein), the longest vein in the body, begins in the medial marginal vein of the dorsum of the foot and ends in the femoral vein about $3 \mathrm{~cm}$. below the inguinal ligament. It ascends in front of the tibial malleolus and along the medial side of the leg in relation with the saphenous nerve. It runs upward behind the medial condyles of the tibia and femur and along the medial side of the thigh and, passing through the fossa ovalis, ends in the femoral vein.

Tributaries.-At the ankle it receives branches from the sole of the foot through the medial marginal vein; in the leg it anastomoses freely with the small saphenous vein, communicates with the anterior and posterior tibial veins and receives many cutaneous veins; in the thigh it communicates with the femoral vein and receives numerous tributaries; those from the medial and posterior parts of the thigh frequently unite to form a large accessory saphenous vein which joins the main vein at a variable level Near the fossa ovalis it is joined by the superficial epigastric, superficial iliac circumflex, and superficial external pudendal veins. A vein, named the thoracoepigastric, runs along the lateral aspect of the trunk between the superficial epigastric vein below and the lateral thoracic vein above and establishes an important communication between the femoral and axillary veins.

The anterior accessory saphenous veins join the upper section of the great saphenous vein in an almost constant position. The anterior accessory saphenous vein is an axial vein which originates from the lateral part of the calf. Then, it ascends to the posterior knee running medially along the thigh to drain into the great saphenous vein in the groin. The anterior accessory saphenous vein almost always connects directly with the great saphenous vein in the confluence of superficial inguinal veins as a sapheno femoral tributary. Rarely, it drains into the great saphenous vein in the upper thigh or directly into a deep vein in the groin. The proximal part runs inside the compartment of the great saphenous vein where it is often separated by a layer of connective tissue. In healthy veins, its course along the thigh is only visible on ultrasound from the groin to where it leaves the fascia or slightly distal of that point. If the vein is refluxive it can be seen varicose directly beneath the skin in several places along its length. As with the thigh tributaries, the exact anatomical course of this vein is superfluous, as it forms part of a venous network. If the anterior accessory saphenous vein is refluxive, it is easily identified because of its superficial location. In the middle third of the thigh, a connecting vein is often found between the anterior accessory saphenous vein and the great saphenous vein. If one of these veins becomes incompetent, the other may become overloaded with reflux from this vein. A good understanding of the venous anatomy is very important and proper evaluation of the venous system is crucial to the appropriate treatment of venous disorders.

\section{Referances:}

1. Labropoulos N, Kang SS, Mansour MA, et al. Primary superficial reflux with competent saphenous trunk. Eur J VascEndovascSurg 1999; 18: 201-206.

2. Garcia-Gimeno $\mathrm{M}$, Rodriguez-Camarero S, TagarroVillalba S, et al. Duplex mapping of 2036 primary varicose veins. J VascSurg 2009; 49: 681-689.

3. Rasmussen LH, Bjoern L, Lawaetz M, et al. Randomised clinical trial comparing endovenous laser ablation with stripping of the great saphenous vein: clinical outcome and recurrence after 2 years. Eur J VascEndovascSurg 2010; 39: 630-635.

4. Bush RG, Flanagan J, Gueldner T, et al. Factors associated with recurrence of varicose veins after thermal ablation: results of the recurrent veins after thermal ablation study. Sci World J 2014; Article ID 505843: 7.

5. Theivacumar NS, Darwood RJ and Gough MJ. Endovenous laser ablation (EVLA) of the anterior accessory great saphenous vein (AAGSV): abolition of sapheno-femoral reflux with preservation of the great saphenous vein. Eur $\mathbf{J}$ VascEndovascSurg 2009; 37: 477-481.

6. Treatment of Varicose Veins. Anthem blue cross blue shield web site, www.anthem.com/medicalpolicies/policies/mp_p w_a053323.htm (accessed 19 May 2014). 